\title{
The Mainz Neutrino Mass Experiment
}

\author{
Ch. Weinheimer*† J. Bonn, B. Bornschein, L. Bornschein, L. Fickinger, B. Flatt, A. \\ Kovalik ${ }^{\ddagger}$ Ch. Kraus, E.W. Otten, J.P. Schall, Th. Thümmler, H. Ulrich \\ Institute of Physics, Joh. Gutenberg University, 55099 Mainz, Germany \\ E-mail: 'weinheimer@iskp.uni-bonn.de'
}

Abstract: The presently lowest limit for the mass of the electron neutrino is $m_{\nu}<$ $2.2 \mathrm{eV} / \mathrm{c}^{2}$ (95 \%C.L.) derived from measurements at Mainz. A proposed experiment KATRIN should improve this neutrino mass sensitivity by one order of magnitude to check the cosmological relevance of neutrino masses and to distinguish between hierarchical and degenerate neutrino mass models.

\section{Introduction}

With the recent evidences for neutrino oscillation and consequently for non-zero neutrino

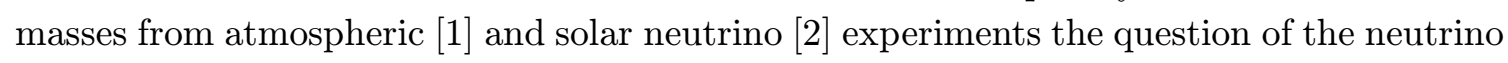
mass scale has become very important, as it has strong consequences for particle physics as well as for astrophysics and cosmology. The investigation of the tritium $\beta$ spectrum near its endpoint measures the mean square of the mass of the "electron neutrino" $m_{\nu_{e}}^{2}=$ $\sum_{j}\left|U_{e j}\right|^{2} m_{j}^{2}$ with neutrino mixing matrix $U$ and neutrino mass eigenstates $m_{j}$; it is the most sensitive of the so-called direct methods and provides information complementary to

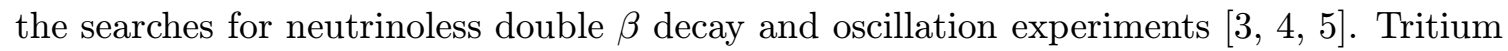
$\beta$ decay is the ideal method to distinguish between models with hierarchical and those with degenerate neutrino masses. Furthermore, neutrino masses up to about $1 \mathrm{eV} / \mathrm{c}^{2}$ are especially interesting for cosmology because of their contribution to the missing hot dark matter in the universe. A mass of about $1 \mathrm{eV} / \mathrm{c}^{2}$ is fitting models of structure formation [6휴].

The tritium $\beta$ decay experiments running at Mainz and Troitsk [i] are currently reaching their sensitivity limit of $2-3 \mathrm{eV}$. Both experiments use spectrometers, which are called MAC-E-Filter (ㅍagnetic $\underline{\text { Adiabatic }}$ Collimation followed by a retarding Electrostatic Filter) $[\overline{9}, 1,1 \overline{0}]$. A future experiment based on this principle, the KArlsruhe TRItium Neutrino experiment (KATRIN) is proposed to improve the neutrino mass sensitivity by one

\footnotetext{
${ }^{*}$ Speaker.

${ }^{\dagger}$ present address: Institut für Strahlen- und Kernphysik, Bonn University, 55115 Bonn, Germany

${ }^{\ddagger}$ on leave from JINR, Dubna
} 
order of magnitude into the sub-eV region. This will allow to check the cosmological relevance of neutrino masses and to distinguish between hierarchical and degenerate neutrino mass scenarios.

\section{The Mainz Neutrino Mass Experiment}

The Mainz experiment uses as $\beta$ source a solid source of molecular tritium, quench condensed on a graphite substrate (HOPG), which is atomic flat on a microscopic scale. The

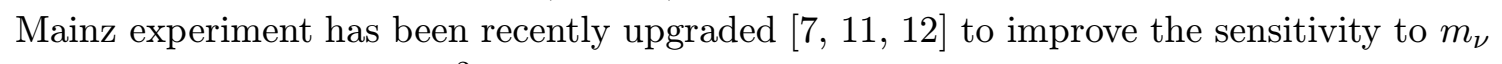
down to a limit of $2 \mathrm{eV} / \mathrm{c}^{2}$ and to check the anomalous excess in the spectrum close to the endpoint reported by the Troitsk group [1] $\left.\overline{7}_{1}, 1,1\right]$. This upgrade allows to use films of a typical thickness of about $480 \AA$, which corresponds to about 140 monolayers. The source diameter is $17 \mathrm{~mm}$ giving a luminosity which is equal to the Troitsk one.

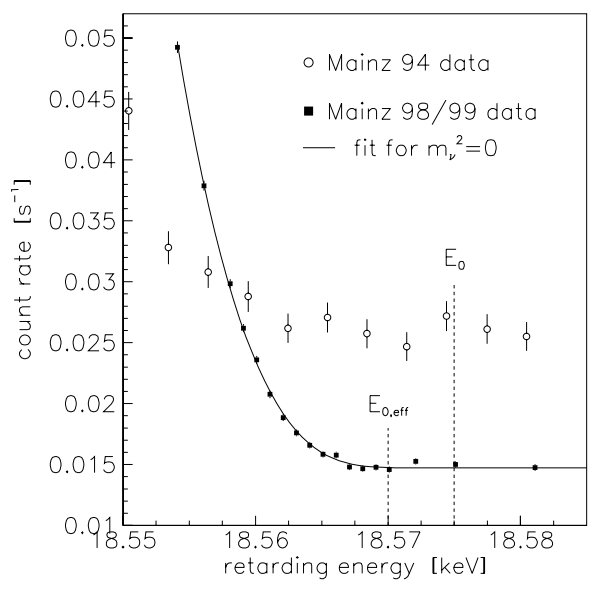

Figure 1: Averaged count rate of the combined 1998 and 1999 data Q3-Q8 (points) with fit (line) in comparison with previous Mainz data from 1994 [1] the retarding energy near the endpoint $E_{0}$, and effective endpoint $E_{0, e f f}$. The latter is width of the filter of $4.8 \mathrm{eV}$ and a mean rovibrational excitation energy of $1.7 \mathrm{eV}$ of the daughter molecule $\left(\mathrm{T}^{3} \mathrm{He}\right)^{+}$. shifted about $5 \mathrm{eV}$ below $E_{0}$ due to the finite

\subsection{The 6 runs of 1998 and 1999}

With the improved setup 6 runs (labelled Q3Q8) of 7 month measurement time in total have been taken in 1998 and 1999. Additional studies on quench condensed $\mathrm{T}_{2}$ films clarified their

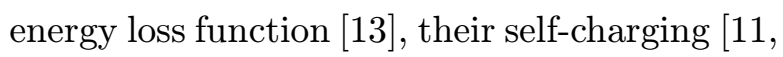
1근, and their dewetting as a function of temperature $\left[\overline{1} \overline{1} \overline{5}_{1}^{1}, \overline{1}, \overline{1} \overline{6}_{1}^{\prime}\right]$.

Fig. ${ }_{1}^{1}$ 's shows the integral $\beta$ spectrum as function of the filter potential for the last $15 \mathrm{eV}$ below the endpoint $E_{0}=18575 \mathrm{eV}$ plus the background region above $E_{0}$. Plotted is the count rate averaged over the runs Q3 to Q8; data obtained in 1994 [1] parison. The improved spectrometer yields a signal to background ratio 10 times better than before and much better statistics has been obtained meanwhile. A fit with $m_{\nu}^{2}$ fixed to zero perfectly fits the new data set over these last $15 \mathrm{eV}$ of the $\beta$ spectrum. This limits any persistent spectral anomaly in this range to an amplitude below $10^{-3} / \mathrm{s}$ (as against a total flux of $10^{8} / \mathrm{s}$ entering the spectrometer). A spectral anomaly, like the fluctuating anomaly reported by the Troitsk group [1] $\left.\overline{7}_{\square}^{\prime}, 8\right]$, on the other hand, reaches amplitudes up to $10^{-2} / \mathrm{s}$.

Figure 12:' shows the fit results on $m_{\nu}^{2}$ with statistical and total uncertainties for the 6 different runs Q3 to Q8 as function of the lower energy limit of the data interval used for the analysis. The monotonous trend towards negative values of $m_{\nu}^{2}$ for larger fit intervals as it was observed for the Mainz 1991 and 1994 data [1] 


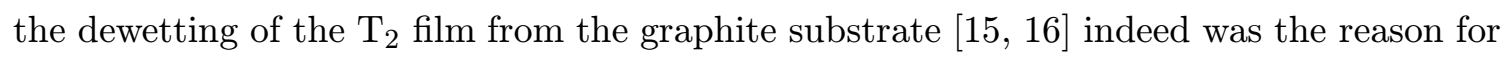
this behavior. Now this effect is safely suppressed at the much lower temperature of the $T_{2}$ film. However, the first two data sets of 1998 (Q3,Q4) still do not fulfill the requirement of being stable with respect to the variation of the lower limit of the fit range and being compatible with the physical allowed range of $m_{\nu}^{2} \geq 0$. But deviations were limited to -10 $\mathrm{eV}^{2} / \mathrm{c}^{4}$ corresponding to a $2 \sigma$ level. The origin of this small residual spectral distortion has not yet been completely clarified, but is certainly connected to a minute instrumental effect depending on running conditions. This statement is corroborated by the fact that the later runs Q5 to Q8 showed absolutely "clean" spectra (see Fig. 2 probably due to a lowering and a stabilization of background rate achieved by applying rf pulses to one of the electrodes of the electrostatic filter in the $2 \mathrm{~s}$ measuring pauses during which the filter potential was changed every $20 \mathrm{~s}$. This way some trapped charges were swept out of the spectrometer, apparently. Trapped particles cause background events through secondary reactions with the residual gas.

Taking the clean data sets Q5-Q8 we use the data down to $70 \mathrm{eV}$ below $E_{0}$. For this interval the combined statistical and systematic uncertainty attains a minimum. The result for $m_{\nu}^{2}$ is

$$
m_{\nu}^{2}=-1.6 \pm 2.5 \pm 2.1 \mathrm{eV}^{2} / \mathrm{c}^{4}
$$

which corresponds to an upper limit of

$$
m_{\nu} \leq 2.2 \mathrm{eV} / \mathrm{c}^{2} \quad(95 \% \text { C.L. })
$$

\subsection{The 2 runs of 2000}

From October to the end of the year 2000 the Mainz group took data. No significant Troitsk-like anomaly was found in particular not in those two subsets of data which were obtained in parallel to data taking at Troitsk (6.12-13.12 and $22.12-28.12)$. But most (not all) of the data subsets suffered again from small residual perturbations which resulted in negative $m_{\nu}^{2}$ fit results in the range of $-10 \mathrm{eV}^{2} / \mathrm{c}^{4}$. We ascribe the reappearance of slight spectral irregularities in our data to a somewhat less favorable status of the spectrometer. Contrary to earlier runs we have not baked it beforehand this time. All our observations leave little doubt that the residual spectral anomalies discussed here are of instrumental origin and connected to the particular electric and magnetic design of MAC-E-Filters, and, moreover, that they can be overcome by paying utmost attention to UHV and surface conditions as well as to the electromagnetic design. ${ }^{1}$

\section{Conclusion and outlook}

The upgraded Mainz experiment has obtained very clean data sets in late 1998 and 1999, which are fully compatible with an ordinary $\beta$ spectrum at neutrino mass zero. The intensive studies of the systematics of quench condensed tritium films allowed to avoid the tritium film roughening and to characterize the film properties with small uncertainties. From the result on $m_{\nu}^{2}$ an upper limit on the neutrino mass of $2.2 \mathrm{eV} / \mathrm{c}^{2}$ is deduced, with is practically the sensitivity limit of the experiment.

\footnotetext{
${ }^{1}$ This statement is supported by the fact that - after having prepared every part of the whole setup in the best way of which we know - our new measurement of October 2001 results in very clean spectra like in late 1998 and 1999.
} 


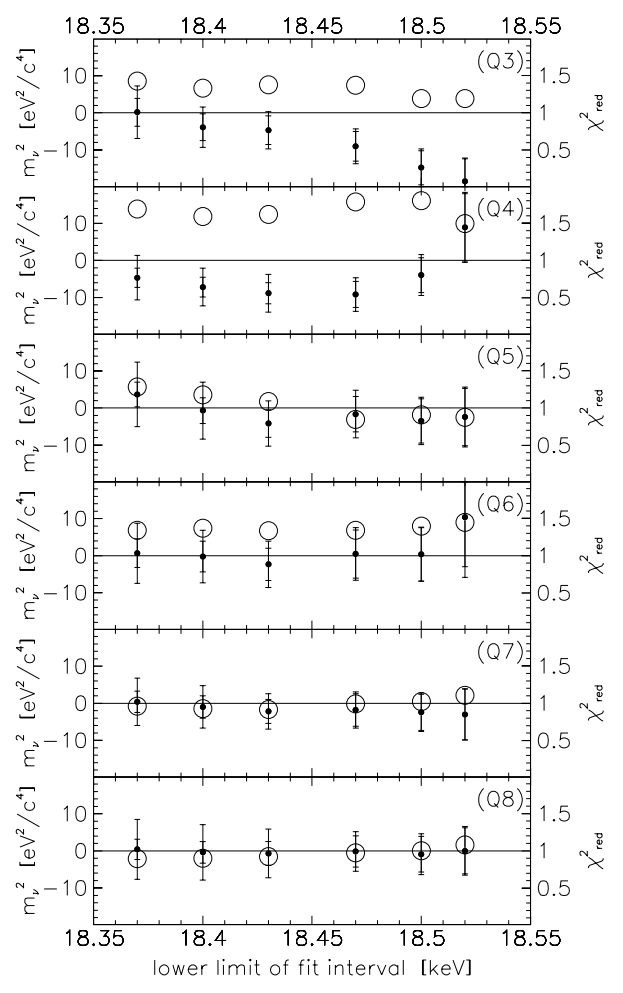

Figure 2: Fit results on $m_{\nu}^{2}$ (left scale, filled circles) for the different runs Q3-Q8 with statistical (inner bars) and total uncertainties (outer bars) in dependence on the lower limit of the fit interval (upper limit: $18.66 \mathrm{keV}$, well above $\left.E_{0}\right)$. The corresponding values of $\chi_{\text {red }}^{2}=\chi^{2} /$ d.o.f. of the fits (open circles) can be read from the right scale.

But this sensitivity is not enough to clarify the open questions concerning the neutrino mass scale and the cosmological role of neutrinos mentioned above. An improvement by another order of magnitude is needed. Currently the only method to reach a sub-eV sensitivity on the neutrino mass is again the investigation of tritium $\beta$ decay: The use of $\beta$ emitters with even lower endpoint energy like Rhenium in combination with cryogenic bolometers is a very important new approach with high potential, but it is still in the development phase much behind present tritium $\beta$ decay experiments. As mentioned above the very important search for neutrinoless double $\beta$ decay gives complementary results and cannot replace the direct searches especially in the case of degenerated neutrino masses $\left[\begin{array}{l}{\left[\begin{array}{l}4 \\ 1\end{array}\right]} \\ ]\end{array}\right]$ Therefore, the most promising and reliable way to reach this sub-eV sensitivity is to utilize the very successful MAC-E-Filter investigating the very upper end of the tritium $\beta$ spectrum.

To clearly reach the sub-eV sensitivity a significantly better energy resolution ( $1 \mathrm{eV}$ instead of $5 \mathrm{eV}$ at Mainz) and a much higher count rate (the region of interest scales with the neutrino mass) is needed, both requiring a significantly larger spectrometer. In a previous paper [i] $\left.{ }_{-}^{\overline{1}} \overline{9}\right]$ the possibility of a spectrometer based on the same MAC-E filter principle but 5 times larger (in linear dimensions) than the present one was investigated. By an additional time-of-flight analysis the spectrometer can transform from an integrating high pass filter into a narrow band filter (MAC-E-TOF mode), an interesting feature for the investigation of systematics [i] $\left.{ }_{1}^{1} \overline{9}\right]$.

Now a strong collaboration with institutions from Fulda/Germany, Karlsruhe/Germany, Mainz Germany, Prague/Chech republic, Seattle/USA and Troitsk/Russia has been formed to carry out the KArlsruhe TRItium Neutrino experiment KATRIN at the German national laboratory Forschungszentrum Karlsruhe. This idea, the motivation and implications for physics was discussed at an international workshop at Bad Liebenzell/Germany in January $2001[2 \overline{0} \overline{0}]$ resulting in a strong support by the international community. Meanwhile a letter of intent is available [20 $\overline{1} \overline{1}]$. KATRIN will built up a large tritium $\beta$ spectrometer with $7 \mathrm{~m}$ diameter and $20 \mathrm{~m}$ length following a pre spectrometer which rejects already $\beta$ electrons except the ones with energies very close to the endpoint. As tritium source a molecular gaseous tritium source will be used, like in the Troitsk experiment but having 
a factor of 40 times higher count rate. A quench condensed molecular tritium source like in Mainz is aimed for additionally as an alternative source. First simulations with quite conservative assumptions show that this experiment should reach an uncertainty on the electron neutrino mass squared $\Delta m_{\nu}^{2}$ of $0.08 \mathrm{eV}^{2} / \mathrm{c}^{4}$. Of course, we are hoping to find a non-zero neutrino mass, but if not this would correspond to an upper limit of $0.35 \mathrm{eV} / \mathrm{c}^{2}$ at $90 \%$ C.L. First funding for KATRIN has been given by the German Bundesministerium für Bildung und Forschung to built a pre-spectrometer and to check new ideas to improve the experiment. A full proposal will be ready within next year, the real construction phase should start in 2003, first data are expected for 2006 .

\section{Acknowledgements}

The work of the Mainz group was supported by the Deutsche Forschungsgemeinschaft under contract Ot33/13 and by the German Bundesministerium für Bildung und Forschung under contract $06 \mathrm{MZ} 866 \mathrm{I} / 5$.

\section{References}

[1] Y. Fukuda, et al., Phys. Rev. Lett. 85 (2000) 3999

[2] Q.R. Ahmad, et al., nucl-ex/0106015

[3] F. Vissani, Nucl.Phys.Proc.Suppl. 100 (2001) 273

[4] Y. Farzan et al., Nucl.Phys. B612 (2001) 59-97

[5] S. Pascoli et al., hep-ph/0110287

[6] M. Tegmark et al., Phys. Rev. D63 (2001) 043007

[7] Ch. Weinheimer et al., Phys. Lett. B460 (1999) 219

[8] V.M. Lobashev, et al, Phys. Lett. B460 (1999) 227

[9] A. Picard et al., Nucl. Inst. Meth. B63 (1992) 345

[10] V.M. Lobashev al., Nucl. Inst. Meth. A240 (1985) 305

[11] H. Barth et al., Prog. Part. Nucl. Phys. 40 (1998) 353

[12] B. Bornschein, $\mathrm{PhD}$ thesis, Mainz university, 2000

[13] V.N. Aseev et al., Eur. Phys. J. D10 (2000) 39

[14] H. Backe et al., Proc. of Neutrino 96, Helsinki, World Scientific/Singapure

[15] L. Fleischmann et al., J. Low Temp Phys. 119 (2000) 615

[16] L. Fleischmann et al., Eur. Phys. J. B16 (2000) 521

[17] A.I. Belesev et al., Phys. Lett. B350 (1995) 263

[18] J. Bonn et al., Nucl. Phys. B (Proc. Suppl.) 91 (2001) 273

[19] J. Bonn et al., Nucl. Inst. and Meth. A421 (1999) 256

[20] International Workshop on "Neutrino Masses in the sub-eV Range" Bad Liebenzell, Germany, January 18-21, 2001, http://www-ik1.fzk.de/tritium/liebenzell/

[21] A. Osipowicz et al., hep-ex/0109033 